Biannual Research Journal Grassroots

Vol.55, No.I, 2021: 232-256

Grassroots

\title{
TERRORISM IN KARACHI, SINDH PAKISTAN: A CASE STUDY OF SAFOORA-INCIDENT
}

\author{
Sanaullah Abbasi \\ Ph.D Scholar \& Inspector General of Police, Khyber Pakhtwun Khwa
}

\begin{abstract}
Terrorism is contentious issue. It has affected the lives of people across the country. This paper analyses the factors for incidence of terrorism in Karachi city which has great economic importance for country. The city represents all communities belonging to various parts of the country. Migration of people from other provinces has significally changed demographic profile of the city. This study presents statistics about various offences committed in Hyderabad and Karachi. It describes important facts about the militant groups involved in the acts of terrorism in Karachi. It has been argued in the study that in some cases the acts of violence were politically motivated and in other cases terrorism acts were sponsored by religious groups.

In order to understand the complex phenomenon of terrorism in Karachi, this study investigates the root causes of terrorism including economic deprivation. In most cases marginal sections of society have been found involved in the acts of terrorism. Thus, this study creates need for social reforms, poverty alleviation and provision of quality education. Further capacity-building of Law Enforcement Agencies to cope with this situation has been emphasized in this study. This paper also recommends some proposals for dealing with the issue of terrorism.
\end{abstract}

Keywords: Terrorism, Economic deprivation, crime, Militant groups, Offences.

\section{INTRODUCTION}

Terrorism has become a serious challenge faced by Pakistan even before the event of $9 / 11,2001$. It is a "home grown" reality with dozens of groups that despite crackdowns on their activities by the law Enforcement Agencies, they continue to flourish while a state of denial and lack of political will allowed security issues to reach their existing critical extent. Ex. Chief of Army Staff Gen. Ashfaque Pervaiz Kiani emphasized the issue by quoting following words: "The strongest motivation for peace through negotiations stems from the state of the economy and the imperative need to divert resources for human development. Military competition, if replaced by economic cooperation, can give hope to millions of South Asians for a-better future" (Karamat, 1999). 
Hard military assets were presumed to be exclusive solution ignoring the soft assets including social / economic cultures culminating and climaxing in Human development index. The nonState actors were the strategic depth in security state paradigm which exists in island of isolation in hostile vicinity neighborhood. Military calculus is not as important as the human development index in vital for any society to grow (Shabana, 2010).

There are two schools of thoughts available for understanding the nature of terrorism:

(1) The "psychological-sociological" school of thought.

(2) The "political-rational" school of thought.

Psychological school emphasizes fear and anxiety as short term and political as long term goal for terrorism and the psychologicalsociological school addresses reliance on psychological condition of individual and peers as group. It depends on profile of an individual terrorist which include paranoia, narcissistic and antisocial behaviour (Million, 1981; Post, 1987).

However, there is no evidence for general personality traits of criminals. Most criminals are having psychological tendencies like activism, thrill and aggression, seeking, an externalist psychological factionalism and mechanism. Characteristics of borderline personalities and narcissistic disorders premeditated thoughts. In this regard, Rational choice theory emphasizes conscious, rational, calculated decision to be opted.

One of the experts on terrorism shared his views that he has been studying terrorists and terrorism for more than twenty years. Yet he still always struck by how disturbingly 'normal' most terrorists seem when one actually sits down and talks to them. He further remarked that many of them are highly articulate and extremely thoughtful individuals for whom terrorism is (or was) an entirely rational choice" (Hoffman, 1998).

However, the two schools may compliment \& supplement one another in-order to explain phenomena of terrorism. 


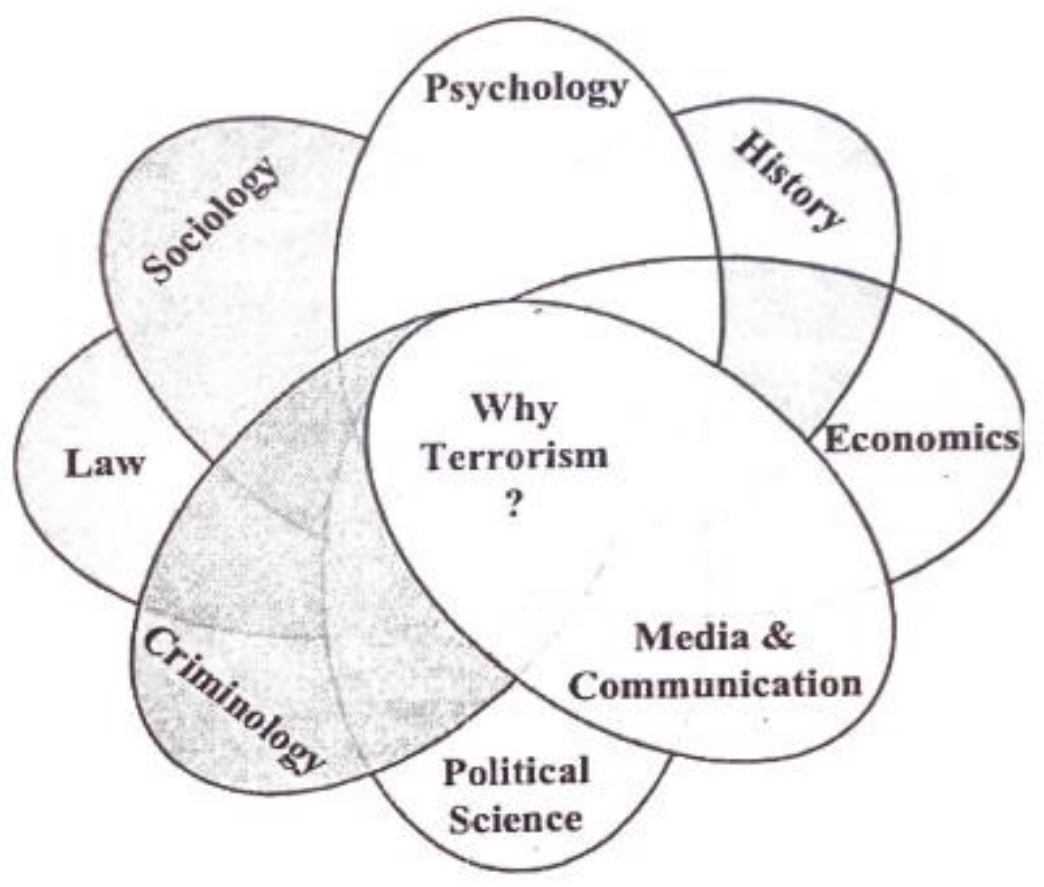

Source: Crenshaw, 1998:386.

\section{PROBLEM STATEMENT}

The menace of terrorism is serious threat to the integrity of Pakistan. Now the threat has grown to the extent that is considered by security apparatus as potential threat to state of Pakistan.

Karachi is the hub of economic activity contributing more than $80 \%$ revenue of the country and the terrorist has targeted Karachi to destabilize Pakistan. If Karachi sneezes, Pakistan catches the cold. Therefore, it is important to study the problem of terrorism in Karachi. Many incidents of terrorism have taken place in Karachi in terms of severity and causalities, which caused serious destruction.

The Safoora incident is case in point which has impacted the entire landscape of Pakistan because the Ismaili Community was targeted which has international connotations.

\section{OBJECTIVES OF STUDY}

1. Explore the socio-economic profile of terrorists of Safoora incident and analyzing facts about this incident. 
2. Explain the perceptions of terrorists with regards to terrorism.

3. Analyze various statistics about offences committed in Hyderabad and Karachi.

\section{RESEARCH METHODOLOGY}

For analysis of the issue of terrorism in Karachi in general and Safoora incidents in particular, the data has been collected through following sources. The Primary data has been collected through indepth interviews of the accused of Safoora incident and the interviews of investigation officers. Besides, the secondary data has been collected books, newspapers, and magazines etc.

\section{SOCIO-ECONOMIC PROFILE OF KARACHI}

The population Karachi is 15 million with an annual growth rate of 4.8 percent (Mujtaba, 2009). The city is considered as the financial and commercial hub of Pakistan, which contributes to $60 \%$ of the country's economy. According to Federal Government, Karachi contributes about 15 percent of the national GDP, 42 percent of value added in manufacturing sector and 25 percent of the revenues of the Federal Government (Mujtaba, 2009).

Karachi is only deep sea port of the country which serves as shipping and maritime center of Pakistan. It serves as major trade route for landlocked countries. 


\begin{tabular}{|c|c|c|}
\hline \multicolumn{3}{|c|}{$\begin{array}{l}\text { TABLE-1 } \\
\text { COMPARISON BETWEEN PLANNED AND UNPLANNED } \\
\text { AREAS OF KARACHI }\end{array}$} \\
\hline Demography & $\begin{array}{l}\text { Planned } \\
\text { Areas }\end{array}$ & $\begin{array}{l}\text { Unplanned } \\
\text { Areas }\end{array}$ \\
\hline a) Average household size & 6.9 & 7.3 \\
\hline b) Gender distribution (male) (\%) & 54 & 65 \\
\hline c) Population (under twenty) & 48.6 & 56.4 \\
\hline d) Crude Birth Rate & 1.3 & 3.6 \\
\hline \multicolumn{3}{|l|}{ Housing } \\
\hline a) Permanent structures $(\%)$ & $70-90$ & 20 \\
\hline b) Semi-permanent structures (\%) & $10-30$ & 19 \\
\hline c) Temporary structures $(\%)$ & - & 5 \\
\hline d) Built Up M2 per person & 19.25 & 11.59 \\
\hline e) Number of persons per room 2 & 0.5 & 303 \\
\hline Access to Utilities & 83 & 50.3 \\
\hline a) Water connections $(\%)$ & 98.4 & 75.8 \\
\hline b) Gas connections $(\%)$ & 75 & 35.1 \\
\hline c) Sewage connections (estimated) (\%) & 85 & 12 \\
\hline \multicolumn{3}{|l|}{ Education } \\
\hline a) Population rate (under ten) & 76 & $48-67$ \\
\hline b) Population with primary education (\%) & 9.5 & 21.7 \\
\hline c) Population with intermediary education (\%) & 19.8 & 16.3 \\
\hline d) Population with Bachelor and above (\%) & 19 & 13.1 \\
\hline
\end{tabular}

\begin{tabular}{|l|l|l|}
\hline a) Population employed (\%) & 65 & 64.7 \\
\hline b) Population unemployed (\%) & 24.9 & 25.3 \\
\hline c) Housewives (\%) & 31 & 34 \\
\hline Income / Expenditure & & \\
\hline a) Average Income (Rs.per month) & $3808-4930$ & $1899-2158$ \\
\hline b) Earned through wages (\%) & 50.8 & 77.7 \\
\hline c) Earned through profit (\%) & 20.2 & 16.8 \\
\hline d) Average expenditure (Rs.per month) & 3083 & $11648-2109$ \\
\hline e) Spent on food (\%) & 53 & 58 \\
\hline f) Spent on rent (\%) & 18 & 13 \\
\hline g) Spent on savings (\%) & 30 & 2.3 \\
\hline Employment & & \\
\hline
\end{tabular}

Source: Arif Hasan, 1996. 
According to Waseem (1998), migration in Karachi was unnatural after partition. He describes four stages of migration to Karcahi. (i) Muhajirs (1940s-50s), (ii) Punjabis and Pakhtuns (1960s80s), (iii) Sindhis (1970s-90s) and (iv) foreigners including Afghans and Iranians.

\section{ETHNO-POLITICAL CONFLICTS}

Politics has changed socio-political dynamics in Sindh. Before 1947 Hindu constituted and urban population but after their migration to India space was created for migrants from India.

In 1950s, Ayub Khoro was annoyed at this state of migration and its impact on demography of Karachi. Formation of sectarian, social and political organizations, especially for the new settlers resulted in polarization between new and old Sindhis.

During martial law of Zia Muhajirs got prominence on the basis of ethnic and language. They were mobilized after Bushra Zadi incident. Resentment and anger among Mohajir community reached at highest. These developments gave rise to the birth of MQM, when General Zia extended quota system for ten more years. Language issue was one of the key factors for creation of MQM.

\section{MILITANTS GROUPS WORKING IN KARACHI}

Jandullah was organized by Atta-ur-Rehman who had deserted Jamate- Muslimeen in 2003. Mr Atta-ur-Rahman was arrested after the attack on Core Commander of Karachi at Clifton Bridge. The command of the, Jundullah was handed over to Qasim Ghori.

Lashkar-e-Jhangvi: Anti-Shia terrorist outfit is also working in Karachi targeting mainly Shia community. Following 4 factions constituted the umbrella L-Jhangvi. (i) Qari Zafar, (ii) Qari Shakeel, (iii) FarooqBangali (iv) Akram Lahori groups. Qari Zafar group has strong linkages with al-Qaeda.

Harkat ul-Mujahideen Al-Alami (HUMA): It was established in 2002 as off shoot of Harakat-ul-Mujhadin. It is mainly Karachi-based organization having operated in Afghanistan and in Kashmir. They tried to target Pervez Musharaf but their attempt was failed. Its leaders Hanif and Imran are in custody \& sentenced to death by the Court.

Sipah-e-sahaba Pakistan (SSP): Sipah-e-Sahaba has Deobund sectarian organization was banned in 2002. However it continued to flourish under the cover names Millat-e-Islamia, Ahle Sunat, wal- 
Jamaat Pakistan (ASWJP), Sipah-e-Sahaba. SSP was established in September 6, 1985 in Jhang, District of Punjab. Maulana Haq Nawaz Jhangvi, Maulana Ziaur Rehman Farooqi, Maulana Eesar-ul-Haq Qasmi and Maulana Azam Tariq were the pioneers of the SSP. The main objectives of Sipah-e-Sahaba (SSP) were as under:

a) To establish Sunni State in Pakistan.

b) To establish the Khilafat system (Khilafat-e-Rashida).

c) To protect the Sunni community and to teheek Shia community.

Sunni Tehreek: Muhammad Salim Qadri, a business man founded Sunni Tehreek in 1990. The ST initially was non-political outfit, focused on four-points:

1) To safeguard the Ahl-e-Sunnat

2) To protect common people from false beliefs

3) Handing over of the Ahl-e-Sunnat mosques and shrines by the administration to the Barelvis.

\section{POLITICALLY-MOTIVATED TERRORISM IN KARACHI}

(Husain, 2010) shows that Zia Martial-Law administrator who staged coup in 1977, faced stiff resistance in Sindh which was strong hold of Zulfiar Ali Bhutto who was hanged to death.

TABLE-2

TERRORISM INCIDENTS, PEOPLE KILLED AND WOUNDED IN PAKISTAN (1974-2007)

\begin{tabular}{|l|l|l|}
\hline Variable & Sum & Mean \\
\hline Incidents & 2590 & 76 \\
\hline Killed & 5840 & 172 \\
\hline Wounded & 11507 & 341 \\
\hline
\end{tabular}

Source: Hussain, 2010.

Hence Bhutto's' Strong Hold in Sindh was serious challenge for Zia. In order to neutralize Bhutto's influence in Sindh, he helped to create Muhajir Qaumi Movement (MQM) (Urdu Speaking urbanbased population)

Kukreja, (2003) Language-related terrorism constituted almost $90 \%$ of terrorism in the cities of Karachi and Hyderabad and $40 \%$ in the country.

Almost $30 \%$ of terrorism in Pakistan is attributed to the Deoband SSP and the Shia groups like Sipah-e-Muhammad (SMP). 
Hence, language and sectarian dynamics explain about $70 \%$ of terrorism in Pakistan.

STATICS OF TERRORISM IN KARACHI

TABLE-3

THIS TABLE SHOWS THE STATICS OF TERRORISM IN KARACHI

\begin{tabular}{|c|c|c|c|}
\hline \multicolumn{4}{|c|}{ Analysis of Target Killing Cases - 2011/2012 } \\
\hline \multicolumn{2}{|c|}{ Political Killing - 2011/2012 } & \multicolumn{2}{|c|}{$\begin{array}{c}\text { Target Killers Arrested } \\
2011 / 2012 \\
\end{array}$} \\
\hline $\begin{array}{c}\text { Party } \\
\text { Affiliation }\end{array}$ & $\begin{array}{l}\text { No. of } \\
\text { Killings }\end{array}$ & $\begin{array}{c}\text { Party } \\
\text { Affiliation }\end{array}$ & $\begin{array}{c}\text { No. of } \\
\text { Accused } \\
\text { Arrested }\end{array}$ \\
\hline ANP & 24 & ANP & 2 \\
\hline $\begin{array}{l}\text { Jamat-e- } \\
\text { Islami }\end{array}$ & 2 & MQM-A & 17 \\
\hline ST & 9 & L.J & 2 \\
\hline MQM-A & 86 & S.M.P & 2 \\
\hline MQM-H & 7 & S.S.P & 2 \\
\hline $\mathrm{PPP} / \mathrm{PSF}$ & 29 & TTP & 9 \\
\hline PAC & 2 & S.T & 8 \\
\hline $\mathrm{KRC}$ & 2 & & \\
\hline OTHERS & 15 & & \\
\hline TOTAL & 176 & TOTAL & 42 \\
\hline
\end{tabular}

Source : CPO Karachi, 2011-12.

This table Show that in these two years maximum number of killed persons was that of the political Killing belongs to MQM Altaf followed by Awami National Party and Pakistan People's Party. During the same period the maximum number of the target killers arrested belonged to MQM Altaf. This shows that there was fight among various political groups. 
TABLE-4

ANALYSIS OF TARGET KILLING CASES

(FROM 1ST JANUARY TO 31ST AUGUST 2013)

\begin{tabular}{|c|c|c|c|c|c|}
\hline \multicolumn{2}{|c|}{ Political Killing- 2013} & \multicolumn{4}{|c|}{ Accused arrested with Affiliation- 2013} \\
\hline \multirow[t]{2}{*}{$\begin{array}{l}\text { Party } \\
\text { Affiliation }\end{array}$} & \multirow[t]{2}{*}{$\begin{array}{l}\text { No. of } \\
\text { Killings }\end{array}$} & \multirow[t]{2}{*}{$\begin{array}{l}\text { Party } \\
\text { Affiliation }\end{array}$} & \multicolumn{2}{|c|}{$\begin{array}{l}\text { No. of Accused } \\
\text { Arrested }\end{array}$} & \multirow[t]{2}{*}{$\begin{array}{l}\text { Total } \\
\text { Arrested }\end{array}$} \\
\hline & & & CID & $\begin{array}{l}\text { Karachi } \\
\text { Police }\end{array}$ & \\
\hline ANP & 9 & MQM-A & 12 & 9 & 21 \\
\hline Jamat-e-Islami & 4 & L.J & 13 & - & 13 \\
\hline Jiye Sindh & 2 & S.T & 3 & - & 3 \\
\hline JUI (F) & 1 & LGW & 9 & 15 & 24 \\
\hline MQM-A & 52 & MQM-H & 1 & - & 1 \\
\hline MQM-H & 3 & SSP & - & 3 & 3 \\
\hline Others & 1 & TTP & - & 11 & 11 \\
\hline PML-N & 4 & ANP & - & 1 & 1 \\
\hline PPP/PSF & 4 & MWM/ISO & - & 9 & 9 \\
\hline PTI & 1 & PPI & - & 3 & 3 \\
\hline S.T & 15 & No Affiliation & 3 & 5 & 8 \\
\hline TOTAL & 96 & TOTAL & 41 & 56 & 97 \\
\hline
\end{tabular}

This Table shows that in 2013 in first 6 months the maximum political killings took place in which MQM Altaf people were killed. The maximum number of target killers belonged to Lashkar-e-Jhangvi followed by MQM Altaf. This shows that either the banned religious outfit has entered into the conflict.

TABLE-5

STRENGTH OF LAW ENFORCEMENT AGENCIES

\begin{tabular}{|l|l|l|}
\hline Police Strength & Sanctioned & $\mathbf{3 0 , 9 5 9}$ \\
\hline & Working & 27,849 \\
\hline & Vacancy & 3,110 \\
\hline Police Ranges & East \\
& West \\
& South \\
\hline Police Districts & 6 \\
\hline Police Sub-Division & 45 \\
\hline Police Stations & 109 \\
\hline
\end{tabular}

Source: CPO Karachi, 2011-12. 
Following is the police population ratio of Karachi in comparison of other mega cities of the world.

TABLE-3

POLICE AND POPULATION RATIO IN KARACHI

\begin{tabular}{|l|l|l|l|}
\hline \multicolumn{1}{|c|}{ CITY } & \multicolumn{1}{|c|}{$\begin{array}{c}\text { Population } \\
\text { (In Million) }\end{array}$} & \multicolumn{1}{c|}{$\begin{array}{c}\text { POLICE } \\
\text { STRENGTH }\end{array}$} & \multicolumn{1}{c|}{ RATIO } \\
\hline London & 7.4 & 48,661 & $1: 152$ \\
\hline Delhi & 16.7 & 57,500 & $1: 291$ \\
\hline New York & 8.2 & 34,500 & $1: 237$ \\
\hline Lahore & 10.9 & 32,600 & $1: 337$ \\
\hline Karachi & 19 & 30,959 & $1: 614$ \\
\hline
\end{tabular}

Source: CPO Karachi, 2011-12.

This show that Karachi police is highly under staffed, it needs more manpower.

\section{BRIEF FACTS OF SAFOORAN INCIDENT}

On 14-05-2015 SIP Ali Akber stated that on 13-05-2015@09: 25 he received a written message from Gadap base that some unknown persons have fired upon a Bus near Al-Azhar Garden and various people have sustained injuries. On receipt of such information the police mobiles and staff of the police station was directed to the place of incident. Superior officers were also informed about the incident. The necessary evidence was collected from the spot. The body of deceased men and women and injuries were already moved to the nearest the Memon Hospital for first aid. After visiting the spot when the police official reached at the Memon Hospital, huge number of dead bodies and injured people were found placed.

On Orders of the higher authorities, the following officers of District Malir reached at Hospital All officers worked together to prepare the memo of inspection of dead bodies of men and women who were dead in the incident of Safooran. Subsequently, the postmartum of deceased were conducted by Dr. Sheeraz, Dr.Aijaz Ahmed, Lady Doctor Roobina Hassan, Lady Doctor Noor-Un-Nissa under the supervision of Surgeon Dr.Jaleel Qadir. After completion of medical process, the dead bodies were handed over to heirs for burial purpose.

From the up to date investigation, 174 Cr.PC. and post martum reports of MLOs and the statements of different people, it was found 
that on 13-05-2015. 55 male /female persons of Agha Khan Ismaili Community boarded on community Bus No. JB- 0333. They were going from Al-Azhar Garden to Aisha Manzil at about 09:10 hours, when the Bus reached at Double Pukhta Road, Anar Garden Scheme No.33 Karachi, about 10 unknown terrorists came on 04 Motorbies and covered by white car containing $4 / 5$ persons, forcibility stopped the Bus and opened: indiscriminate fires with automatic weapons with intention to kill people. As a result 27 male and 18 female were killed and 8 sustained severe injuries. Due to the firing bus was damaged. The terrorists were seen by the number of pedestrian and the persons of vicinity before and after the incident.

The act of these unknown terrorists, helpers, associates, facilitators for killing of both male / female and causing injury and damaging the Bus is tribal $\mathrm{u} / \mathrm{s} 302 / 324 / 427 / 109 / 34$. Hence the case against the unknown terrorists was registered.

\begin{tabular}{|l|l|l|}
\hline \multicolumn{4}{|c|}{ FIR NO.195/2015 PS SACHAL DISTRICT MALIR KARACHI, } \\
DATED: 13.05.2015 @ 0910 HOURS \\
\hline 1 & \multicolumn{3}{|c|}{ Date \& Time of Report } & $14.05 .2015 @$ @ 2300 hours \\
\hline 2 & $\begin{array}{l}\text { Name \& Address (Report } \\
\text { No.43) }\end{array}$ & $\begin{array}{l}\text { SIP Ali Akber of PS Sachal District } \\
\text { Malir Karachi. }\end{array}$ \\
\hline 3 & Brief condition (With U/S) & 302/324/427/109/34 R/W ATA-7 \\
\hline 4 & $\begin{array}{l}\text { Jurisdiction where incident } \\
\text { occurred }\end{array}$ & $\begin{array}{l}\text { Double Pukhta Road near Anar Garden } \\
\text { Scheme 33 Malir Karachi. with the } \\
\text { jurisdiction North East from PS Sachal } \\
\text { distance 2.5 Kilometer. }\end{array}$ \\
\hline 5 & $\begin{array}{l}\text { If any message received } \\
\text { concerned PS or Investigation } \\
\text { made by SIO on the spot } \\
\text { received or otherwise }\end{array}$ & $\begin{array}{l}\text { The message convey to the community } \\
\text { people and their relatives to registered } \\
\text { FIR against the terrorists but they did not } \\
\text { response subsequently the Govt. has } \\
\text { taken the action and registered the case } \\
\text { FIR. }\end{array}$ \\
\hline 6 & $\begin{array}{l}\text { Date \& Time of Departure } \\
\text { from PS }\end{array}$ & By Doc on 14.05.2015 \\
\hline
\end{tabular}

Source: Police Record, 2015.

\section{VARIOUS OFFENCES}

ATTACK ON POLICE MOBILE-2013: In the month of December 2013, a police mobile / escort of Mirza Yousuf (Shia Aalim) were parked near Farhan Apartment. Following terrorists started indiscriminate firing upon mobile. Two police officials were martyred due to firing. Terrorist used $9 \mathrm{~mm}$ pistols. 
Biannual Research Journal Grassroots Vol.55, No.I: 232-256

\begin{tabular}{|l|l|l|l|l|l|}
\hline S\# & $\begin{array}{l}\text { FIR NO } \\
\text { \& U/S }\end{array}$ & $\begin{array}{l}\text { DATE OF } \\
\text { OFFENCE }\end{array}$ & \multicolumn{1}{|c|}{ ARRESTED } & $\begin{array}{l}\text { NOT } \\
\text { ARRESTED }\end{array}$ & $\begin{array}{l}\text { POLICE } \\
\text { STATION }\end{array}$ \\
\hline & $855 / 13$ & $28-12-13$ & i) Tahir & i) Umer @ & Shahra \\
& u/s & at 2115 & Hussain & Jawad @ & -e- \\
& $302 / 34$ & & Minhas @ & ii) Amir@ \\
PPC & & Uncle & Faisal \\
& ii) Saad Aziz & $\begin{array}{l}\text { iii) Imran } \\
\text { Gujjar @ } \\
\text { 7-ATA }\end{array}$ & & @ Tin Tin & \\
& & & Bhatti & \\
\hline
\end{tabular}

Source: Police Record, 2013.

FIRING ON POLICE MOBILE AT LATIFABAD HYDERABAD- 2014: In the month of Aug 2014, terrorist along with other accused persons attacked upon police mobile with deadly fire arms in the limit of PS A Section Latifabad resultantly three police personnel namely (i) PC Masood Zafar (2) PC Raashid Ali (3) PC Waseem embraced Shahadat and PC David was injured.

\begin{tabular}{|c|c|c|c|c|c|}
\hline S\# & FIR NO \& U/S & $\begin{array}{l}\text { DATE OF } \\
\text { OFFENCE }\end{array}$ & ARRESTED & $\begin{array}{l}\text { NOT } \\
\text { ARRESTED }\end{array}$ & $\begin{array}{l}\text { POLICE } \\
\text { STATION }\end{array}$ \\
\hline & $\begin{array}{l}152 / 2014 \mathrm{u} / \mathrm{s} \\
302 / 324 / 353 \\
/ 427 / 34 \quad \mathrm{PPC} \\
\mathrm{r} / \mathrm{w} 6 / 7-A T A\end{array}$ & $\begin{array}{l}06-07- \\
2014 \\
\text { at } \\
2330\end{array}$ & $\begin{array}{l}\text { i) Tahir } \\
\text { Hussain } \\
\text { Minhas @ } \\
\text { Uncle } \\
\text { Sain } \\
\text { iii) Saad Aziz } \\
\text { a, Tin Tin }\end{array}$ & $\begin{array}{l}\text { i) Umer @ } \\
\text { Jawad } \\
\text { ii) Amir@ @ } \\
\text { Abbas @ } \\
\text { Yameen } \\
\text { ii) Sharif } \\
\text { (Hyd) }\end{array}$ & $\begin{array}{l}\text { A Section } \\
\text { Latifabad }\end{array}$ \\
\hline
\end{tabular}

Source: Police Record, 2014.

HAND GRENADE ATTACK ON POLICE MOBILE NEAR SAREENA MARKET-2014: In the year 2014, Saad Aziz was directed to throw hand grenade on a police mobile parked near Sareena Market. The hand grenade and motorcycle were provided to him by Umer@ Haider Abbas. Saad Aziz did so. He (Tahir Minhas) was sitting in a car at that time. PC Tahir Farooq was injured in this incident). 
Biannual Research Journal Grassroots Vol.55, No.I: 232-256

\begin{tabular}{|l|l|l|l|l|l|}
\hline $\begin{array}{l}\text { S } \\
\#\end{array}$ & FIR NO \& U/S & $\begin{array}{l}\text { DATE OF } \\
\text { OFFENCE }\end{array}$ & ARRESTED & $\begin{array}{l}\text { NOT } \\
\text { ARRESTED }\end{array}$ & $\begin{array}{l}\text { POLICE } \\
\text { STATION }\end{array}$ \\
\hline & $367 / 2014 \mathrm{u} / \mathrm{s}$ & $13-09-14$ & i) Tahir & i) Umer @ & Temoria \\
& $324 / 427 / 34$ & At 2115 & Hussain & Jawad @ & \\
& r/w 3/4 Ex & & $\begin{array}{l}\text { Minhas @ } \\
\text { Abbas }\end{array}$ & \\
Act r/w 7- & & Uncle @ & & \\
ATA Sain & & iii) Saad & & \\
& & & Aziz @ Tin & & \\
& & & Tin & & \\
\hline
\end{tabular}

Source: Police Record, 2014.

DACOITY IN ABL HEERABD HYDERABAD- 2014: In the month of Oct 2014, he along with other accused persons committed dacoity in ABL Bank Heerabad Hyderabad and looted 25 lacs from the Bank.

\begin{tabular}{|c|c|c|c|c|c|}
\hline $\begin{array}{l}\mathbf{S} \\
\#\end{array}$ & FIR NO \& U/S & $\begin{array}{l}\text { DATE OF } \\
\text { OFFENCE }\end{array}$ & ARRESTED & $\begin{array}{l}\text { NOT } \\
\text { ARRESTED }\end{array}$ & $\begin{array}{l}\text { POLICE } \\
\text { STATION }\end{array}$ \\
\hline & $\begin{array}{l}195 / 2014 \mathrm{u} / \mathrm{s} \\
394 / 395 / 353 / 34 \\
2 \\
\text { PPC }\end{array}$ & $\begin{array}{l}20-10-14 \\
\text { at } 0940\end{array}$ & $\begin{array}{l}\text { i) Tahir } \\
\text { Hussain } \\
\text { Minhas@ @ } \\
\text { Uncle @ } \\
\text { Sain Saad } \\
\text { ii) Sziz@ Tin } \\
\text { Tin } \\
\text { iii) Asad- } \\
\text { ur-Rehman } \\
\text { @ } \\
\text { Malik }\end{array}$ & $\begin{array}{l}\text { i) Zaheer @ } \\
\text { Chashmato } \\
\text { ii) Amir @ } \\
\text { Abbas @ } \\
\text { Yameen } \\
\text { iii)Imran @ } \\
\text { Bhatti @ } \\
\text { Salman } \\
\text { iv) Sharif- } \\
\text { Hyd } \\
\text { v) Talha } \\
\text { (Killed } \\
\text { Hyd) vi) } \\
\text { Mistari } \\
\text { Pathan vii) } \\
\text { Sharmeela } \\
\text { Pathan }\end{array}$ & Market \\
\hline
\end{tabular}

Source: Police Record, 2014.

FIRING ON PREEDY POLICE MOBILE - 2015: In the month of March 2015, he along-with other accused persons riding on motorcycles went to M.A Jinnah Road where they fired upon the police officials sitting in police mobile. In this attack three police officials namely (1) ASI Riazat Ali Shah (2) HC Rashid Ali \& (3) PC Saeed embraced Shahdat. 
Biannual Research Journal Grassroots Vol.55, No.I: 232-256

\begin{tabular}{|c|c|c|c|c|c|}
\hline S\# & $\begin{array}{l}\text { FIR NO \& } \\
\text { U/S }\end{array}$ & $\begin{array}{l}\text { DATE OF } \\
\text { OFFENCE }\end{array}$ & ARRESTED & NOT ARRESTED & $\begin{array}{l}\text { POLICE } \\
\text { STATION }\end{array}$ \\
\hline & $\begin{array}{l}231 / 2015 \mathrm{u} / \mathrm{s} \\
302 / 324 / 353 \\
/ 34 \mathrm{PPC} \mathrm{r} / \mathrm{w} \\
\text { 7-ATA }\end{array}$ & $\begin{array}{l}10-03-15 \\
\text { at } 2200\end{array}$ & $\begin{array}{l}\text { i) Tahir } \\
\text { Hussain } \\
\text { Minhas @ } \\
\text { Uncle @ Sain } \\
\text { ii) Saad Aziz } \\
\text { @ Tin Tin } \\
\text { iii) Asad-ur- } \\
\text { Rehman @ } \\
\text { Malik }\end{array}$ & $\begin{array}{l}\text { i) Umer @ } \\
\text { Hafiz } \\
\text { Jawad } \\
\text { ii) Abdul } \\
\text { Rehman @ } \\
\text { Leo King @ } \\
\text { Choto } \\
\text { iii) Tayyab }\end{array}$ & Preedy \\
\hline
\end{tabular}

Source: Police Record, 2015.

FIRING ON POLICE OFFICIALS AT PAKISTAN CHOWK-2015: The same night after firing upon police mobile they went towards Pakistan Chowk where two police constables were standing by road side. They parked their motorcycles by road side, went near the police constables and killed them by firing with pistol. In this incident (i) HC Shakeel \& (ii) PC Mujaheed Hussain were martyred.

\begin{tabular}{|l|l|l|l|l|l|}
\hline S\# & FIR NO \& U/S & $\begin{array}{l}\text { DATE OF } \\
\text { OFFENCE }\end{array}$ & ARRESTED & $\begin{array}{l}\text { NOT } \\
\text { ARRESTED }\end{array}$ & $\begin{array}{l}\text { POLICE } \\
\text { STATION }\end{array}$ \\
\hline & $120 / 2015 \mathrm{u} / \mathrm{s}$ & $10-03-15$ & i) Tahir & i) Umer @ & Aram \\
& $302 / 324 / 353 /$ & at 2112 & Hussain & Hafiz @ & Bagh \\
& 34 PPC r/w & & Minhas @ & Jawad & \\
& $7-$ ATA & & $\begin{array}{l}\text { Uncle @ } \\
\text { Sain Ali }\end{array}$ & Rehman @ & \\
& & ii) Saad Aziz & Tona @ & \\
& & @ Tin Tin i) & Choto iii) & \\
& & Asad-ur- & Tayyab & \\
& & Rehman @ & & \\
& & & Malik & & \\
\hline
\end{tabular}

Source: Police Record, 2015.

BLAST AT BHORI MOSQUE ARAM BAGH-2015: In the month of March 2015, on his directives Saad Aziz and other accused persons conducted motorcycles IED blast at Bhori Mosque Aram Bagh. Abdul Mansoori Pathanhad arranged the IED fitted motorcycle and also detonated the IED with remote. Saad Aziz made video of the blast and handed over the memory card to Umer@ Haider Abbas. In this incident two persons namely (i) Shabir Bhai \& (ii) Hassnain were 
Biannual Research Journal Grassroots Vol.55, No.I: 232-256

killed whereas (i) Shabir Saifullah (ii) Abdullah (iii)Talha \& (iv) Daniyal of Bhori Community were injured.

\begin{tabular}{|c|c|c|c|c|c|}
\hline S\# & FIR NO \& U/S & $\begin{array}{l}\text { DATE OF } \\
\text { OFFENCE }\end{array}$ & ARRESTED & $\begin{array}{l}\text { NOT } \\
\text { ARRESTED }\end{array}$ & $\begin{array}{l}\text { POLICE } \\
\text { STATION }\end{array}$ \\
\hline & $\begin{array}{l}147 / 215 \mathrm{u} / \mathrm{s} \\
302 / 324 / 427 / \\
34 \mathrm{PPC} \mathrm{r/w} \\
3 / 4 \\
\text { Exp Act r/w 7- } \\
\text { ATA }\end{array}$ & $\begin{array}{l}20-03-15 \\
\text { at } 1350\end{array}$ & $\begin{array}{l}\text { i) Tahir } \\
\text { Hussain } \\
\text { Minhas @ } \\
\text { Uncle @ Sain } \\
\text { ii) Saad Aziz } \\
\text { @ Tin Tin }\end{array}$ & $\begin{array}{l}\text { 1) Umer@ } \\
\text { Haider } \\
\text { Abbas } \\
\text { ii) Abdullah } \\
\text { Mansoori } \\
\text { iii) Tayyab }\end{array}$ & $\begin{array}{l}\text { Aram } \\
\text { Bagh }\end{array}$ \\
\hline
\end{tabular}

\begin{tabular}{|c|c|c|c|c|c|}
\hline S\# & $\begin{array}{l}\text { FIR NO \& } \\
\text { U/S }\end{array}$ & $\begin{array}{l}\text { DATE OF } \\
\text { OFFENCE }\end{array}$ & ARRESTED & $\begin{array}{l}\text { NOT } \\
\text { ARRESTED }\end{array}$ & $\begin{array}{l}\text { POLICE } \\
\text { STATION }\end{array}$ \\
\hline & $\begin{array}{l}\text { 195/2015 u/s } \\
302 / 324 / 427 / \\
109 / 34 P P C \\
\text { r/w } \\
\text { 7-ATA }\end{array}$ & $\begin{array}{l}13-05-15 \\
\text { at } 0910\end{array}$ & $\begin{array}{l}\text { i) Tahir } \\
\text { Hussain } \\
\text { Minhas @ } \\
\text { Uncle@ Sain } \\
\text { ii) Saad Aziz } \\
\text { @ Tin Tin } \\
\text { iii) Asad-ur- } \\
\text { Rehman @ } \\
\text { Malik }\end{array}$ & $\begin{array}{l}\text { i) Umer @ } \\
\text { Haider } \\
\text { Abbas } \\
\text { ii) Tayyab @ } \\
\text { Like Blue iii) } \\
\text { All Rehman } \\
\text { @ } \\
\text { Tona @ } \\
\text { Choto iv) } \\
\text { Abdullah } \\
\text { Mansoori } \\
\text { (Pathan) } \\
\text { v) Mistari } \\
\text { Pathan } \\
\text { vi) } \\
\text { Unknown } \\
\text { Pathan vii) } \\
\text { Unknown } \\
\text { Pathan viii) } \\
\text { Dawood } \\
\text { ix) } \\
\text { Mehmood } \\
\text { x) Abdullah } \\
\text { Yousuf }\end{array}$ & $\begin{array}{l}\text { Sachal } \\
\text { District } \\
\text { Malir }\end{array}$ \\
\hline
\end{tabular}

Source: Police Record, 2015.

\section{OTHER DISCLOSERS}

MURDER OF CH. SOHAIL AT HYDERABAD-2010: In the end of year 2011 Azeem Ahmed Sheikh @ Asif \& Farhan killed one Sohail employee of FIAat Latifabad No.8 Hyderabad on suspicion of being informer. Later on they informed the police official about this killing. 
Biannual Research Journal Grassroots Vol.55, No.I: 232-256

\begin{tabular}{|c|c|c|c|c|c|}
\hline S\# & $\begin{array}{l}\text { FIR NO \& } \\
\text { U/S }\end{array}$ & $\begin{array}{l}\text { DATE OF } \\
\text { OFFENCE }\end{array}$ & ARRESTED & $\begin{array}{l}\text { NOT } \\
\text { ARRESTED }\end{array}$ & $\begin{array}{l}\text { POLICE } \\
\text { STATION }\end{array}$ \\
\hline & $\begin{array}{l}275 / 2010 \\
\mathrm{u} / \mathrm{s} \\
302 \text { PPC }\end{array}$ & $\begin{array}{l}29-10- \\
2010 \text { at } \\
1230 \\
\text { to } 1430\end{array}$ & $\begin{array}{l}\text { i) Azeem } \\
\text { Sheikh @ } \\
\text { Asif } \\
\text { ii)Farhan }\end{array}$ & NIL & $\begin{array}{l}\text { Section A } \\
\text { Latifabad. }\end{array}$ \\
\hline
\end{tabular}

Source: Police Record, 2010.

MURDER OF St MANOJ KUMAR AT HYDERABAD-2010: In the year 2010 Azeem Ahmed Sheikh @ Asif \& Farhan killed SI Manoj Kumarnear Makki Masjid Heerabad Hyderabad who was performing duty at Market Police Station. Later on they informed me about this case.

\begin{tabular}{|l|l|l|l|l|l|}
\hline S\# & $\begin{array}{l}\text { FIR NO \& } \\
\text { U/S }\end{array}$ & $\begin{array}{l}\text { DATE OF } \\
\text { OFFENCE }\end{array}$ & ARRESTED & $\begin{array}{l}\text { NOT } \\
\text { ARRESTED }\end{array}$ & $\begin{array}{l}\text { POLICE } \\
\text { STATION }\end{array}$ \\
\hline & $283 / 2010$ & & i) Azeem & i) Shahmim, & Market \\
& u/s & & Ahmed & & Hyderab \\
& $302 / 353 / 34$ & & Sheikh @ & & ad \\
& PPC r/w 6/7 & & Asif & & \\
ATA & & ii) Farhan & & \\
\hline
\end{tabular}

MURDER OF MQM WORKER ASIM ZAIDI AT HYDERABAD-2011: In the year 2011 Azeem Ahmed Sheikh @ Asif \& Farhan killed Asim Zaidi worker of MQM Hyderabad at Hill Top Thandi Sarak Hyderabad. Asim Zaidi has business of cable at Latifabad No.7, who some time ago has abused a Muslim scholar at Latifabad No.8 Hyderabad. Later on they informed me about this case.

\begin{tabular}{|l|l|l|l|l|l|}
\hline S\# & $\begin{array}{l}\text { FIR NO \& } \\
\text { U/S }\end{array}$ & $\begin{array}{l}\text { DATE OF } \\
\text { OFFENCE }\end{array}$ & ARRESTED & $\begin{array}{l}\text { NOT } \\
\text { ARRESTED }\end{array}$ & $\begin{array}{l}\text { POLICE } \\
\text { STATION }\end{array}$ \\
\hline $\begin{array}{l}03 / 2011 \\
\text { u/s302/34 } \\
\text { PPC Q.Ord }\end{array}$ & $\begin{array}{l}08-01-11 \\
\text { at } 1433\end{array}$ & $\begin{array}{l}\text { i) Azeem } \\
\text { Ahmed Sheikh } \\
\text { @ Asif } \\
\text { ii) Farhan }\end{array}$ & & $\begin{array}{l}\text { Makki } \\
\text { Shah }\end{array}$ \\
\end{tabular}

Source: Police Record, 2011.

MURDER OF POLICE SI ADEEL AHMED KHAN NEAR RADO APARTMENT-2013: In the year 2013 Umer@ Hafiz@ Jawad, Tahir Pathan, Imran Gujjar@ Bhatti went to Gulistan-e-Johar on two motorcycles. Imran Gujjar@ Bhatti \& Tahir fired upon a police man with $9 \mathrm{~mm}$ pistols who was sitting near Rado Apartment and killed 
him. Target was given by Hamza. Later on they informed me about this incident.

\begin{tabular}{|c|c|c|c|c|c|}
\hline S\# & $\begin{array}{l}\text { FIR NO \& } \\
\text { U/S }\end{array}$ & $\begin{array}{l}\text { DATE OF } \\
\text { OFFENCE }\end{array}$ & ARRESTED & $\begin{array}{l}\text { NOT } \\
\text { ARRESTED }\end{array}$ & $\begin{array}{l}\text { POLICE } \\
\text { STATION }\end{array}$ \\
\hline & $\begin{array}{l}347 / 2013 \\
\mathrm{u} / \mathrm{s} \\
302 / 34 \\
\mathrm{PPC}\end{array}$ & $\begin{array}{l}15-06-13 \\
\text { at } 2315\end{array}$ & & $\begin{array}{l}\text { i) Umer@ } \\
\text { Hafiz @ } \\
\text { Jawad } \\
\text { ii) Tahir } \\
\text { Pathan } \\
\text { iii) Imran } \\
\text { Gujjar@ } \\
\text { Bhatti } \\
\text { iv) Sarfaraz } \\
\text { @ } \\
\text { Hamza }\end{array}$ & $\begin{array}{l}\text { Shahreh- } \\
\text { e-Faisal }\end{array}$ \\
\hline
\end{tabular}

Source: Police Record, 2013.

BOMB BLAST AT LANDHI- 2013: Few days before the general election 2013, Imran Gujjar@ Bhatti \& Zaheer@ Chashmato put a bomb on the roof of cabin at Landhi which was blasted through mobile phone device. Later on they informed me about this incident.

\begin{tabular}{|l|l|l|l|l|l|}
\hline S\# & FIR NO \& U/S & $\begin{array}{l}\text { DATE OF } \\
\text { OFFENCE }\end{array}$ & ARRESTED & $\begin{array}{l}\text { NOT } \\
\text { ARRESTED }\end{array}$ & $\begin{array}{l}\text { POLICE } \\
\text { STATION }\end{array}$ \\
\hline & $47 / 2013 \mathrm{u} / \mathrm{s}$ & & & i) Imran Gujjar & Landhi \\
& $302 / 324 / 427 / 3$ & & & @ Bhatti & \\
& 4 PPC 3/4 & & & ii) Zaheer @ & \\
& ExpAct r/w 7- & & & Chashmato & \\
ATA & & $\begin{array}{l}\text { iii) Zakir } \\
\text { (Behari) }\end{array}$ & \\
& & & c/o Imran & \\
& & & Gujjar & \\
& & & &
\end{tabular}

Source: Police Record, 2013.

KILLING OF POLICE OFFICIAL AT LATIFABAD HYDERABAD2013: In the month of Sep 2013, Chashmato \& Imran Bhatti and other accused killed a police man at Latifabad No.6 near Mehmood Gardens Hyderabad. Later on they informed me about this incident. 
Biannual Research Journal Grassroots Vol.55, No.I: 232-256

\begin{tabular}{|l|l|l|l|l|l|}
\hline S\# & $\begin{array}{l}\text { FIR NO } \\
\text { \& U/S }\end{array}$ & $\begin{array}{l}\text { DATE OF } \\
\text { OFFENCE }\end{array}$ & ARRESTED & NOT ARRESTED & $\begin{array}{l}\text { POLICE } \\
\text { STATION }\end{array}$ \\
\hline & & & & i) Zaheer @ & \\
& & & & Chashmato & \\
& & & ii) Imran Gujjar @ & \\
& & & Bhatti & \\
& & & iii) Talha c/o & \\
& & & lmran Gujjar & \\
& & & (Killed Hyd) & \\
& & & iv) Amir @ Abbas & \\
\hline
\end{tabular}

Source: Police Record, 2013.

KILLING OF SI NAZEER AT SHAH FAISAL COLONY-2014: In the month of Jan 2014, Sarfaraz @ Hamza along-with others accused persons killed a Si at Shah Faisal Colony Karachi.

\begin{tabular}{|l|l|l|l|l|l|}
\hline S\# & FIR NO \& & DATE OF & ARRESTED & NOT & POLICE \\
URRESTED & ORTATION \\
\hline & $25 / 2014$ & $22-01-14$ & & i) Sarfaraz @ & Shah \\
& u/s 02/34 & at 2000 & & Hamza & Faisal \\
& r/w 7- & & & ii) Umer @ & Colony \\
ATA & & & Hafiz@ & \\
& & & Jawad & \\
& & & iii) Amir @ & \\
& & & Abbas & \\
& & & iv) Imran & \\
\hline
\end{tabular}

Source: Police Record, 2014.

SUICIDAL ATTACK ON RANGER CONVOY-2014: In the month of Jan 2014, Imran Bhatti @ other accused person and a suicidal bomber went to Qayyumabad where the suicidal bomber blown himself away near the Rangers Convoy.

\begin{tabular}{|c|c|c|c|c|c|}
\hline S\# & $\begin{array}{l}\text { FIR NO \& } \\
\text { U/S }\end{array}$ & $\begin{array}{l}\text { DATE OF } \\
\text { OFFENCE }\end{array}$ & ARRESTED & $\begin{array}{l}\text { NOT } \\
\text { ARRESTED }\end{array}$ & $\begin{array}{l}\text { POLICE } \\
\text { STATION }\end{array}$ \\
\hline & $\begin{array}{l}95 / 2014 \quad \mathrm{u} / \mathrm{s} \\
324 / 427 / 34 \\
\text { PPC r/w } 3 / 4 \\
\text { Exp Act r/w } \\
7- \\
\text { ATA }\end{array}$ & $\begin{array}{l}14-02-14 \\
\text { at } 0920\end{array}$ & & $\begin{array}{l}\text { i) Umer @ } \\
\text { Hafiz @ } \\
\text { Jawad Imran } \\
\text { ii) } \\
\text { Bhatti } \\
\text { (provide } \\
\text { suicidal } \\
\text { jacket) } \\
\text { iii) Anas } \\
\text { (provided } \\
\text { suicidal) }\end{array}$ & $\begin{array}{l}\text { Korangi } \\
\text { Industrial } \\
\text { Area } \\
\text { (KIA) }\end{array}$ \\
\hline
\end{tabular}

Source: Police Record, 2014. 
HAND GRENADE ATTACK AT AYESHA MANZIL IMAM BARGAH2014: In the month of Oct 2014, Umer@ Jawad \& other accused persons thrown hand grenade on the Ayesha Manzil Imam Bargah/Islamic Research Centre in which one chilled Ahsan Batool was killed. Later on they informed me about this incident.

\begin{tabular}{|l|l|l|l|l|l|}
\hline S\# & $\begin{array}{l}\text { FIR NO \& } \\
\text { U/S }\end{array}$ & $\begin{array}{l}\text { DATE OF } \\
\text { OFFENCE }\end{array}$ & ARRESTED & $\begin{array}{l}\text { NOT } \\
\text { ARRESTED }\end{array}$ & $\begin{array}{l}\text { POLICE } \\
\text { STATION }\end{array}$ \\
\hline & $244 / 2014$ & $28-10-14$ & i) Saad Aziz & i) Imran & Gulberg \\
& u/s & at 2115 & @ Tin Tin & Bhatti @ & \\
$302 / 324 / 34$ & & & Salman & \\
& & & ii) Sharmeela & \\
PPC r/w 3/4 & & $\begin{array}{l}\text { Pathan } \\
\text { Exp Act r/w }\end{array}$ & & iii) Ali Reh \\
7-ATA & & & man @ Tona & \\
& & & @ Choto & \\
& & & & \\
\hline
\end{tabular}

Source: Police Record, 2014.

HAND GRENADE ATTACK NEAR KAMRAN CHORANGI IMAM BARGAH-2014: In the month of Oct 2014, Umer@ Jawad \& other accused person thrown hand grenade at the Masjid \& Imam Bargah Wahat Muslimen Kamran Chorangi Gulistan-e-Johar Later on they informed me about this incident (FIR not lodged).

HAND GRENADE ATTACK ON POLICE MOBILE AT NIPA CHORANGI-2014: In the month of Oct 2014, Saad Aziz \& other accused person attacked a police mobile with hand grenade at Nipa Chorangi. The mobile was damaged (burnt). Later on they informed me about this incident.

\begin{tabular}{|c|c|c|c|c|c|}
\hline S\# & FIR NO \& U/S & $\begin{array}{l}\text { DATE OF } \\
\text { OFFENCE }\end{array}$ & ARRESTED & $\begin{array}{l}\text { NOT } \\
\text { ARRESTED }\end{array}$ & $\begin{array}{l}\text { POLICE } \\
\text { STATION }\end{array}$ \\
\hline & $\begin{array}{l}526 / 2014 \quad \mathrm{u} / \mathrm{s} \\
353 / 324 / 435 / \\
34 \text { PPC r/w 3/4 } \\
\text { Exp Act r/w } \\
\text { 7-ATA }\end{array}$ & $\begin{array}{l}31-10-14 \\
\text { at } 2030\end{array}$ & $\begin{array}{l}\text { i) Saad Aziz } \\
\text { (a) Tin Tin }\end{array}$ & $\begin{array}{l}\text { i) Umer @ } \\
\text { Hafiz@ } \\
\text { Jawad } \\
\text { ii) All } \\
\text { Rehman @ } \\
\text { Tona @ } \\
\text { Choto } \\
\text { iii) } \\
\text { Sharmeela } \\
\text { Pathan } \\
\text { iv) Sabir }\end{array}$ & $\begin{array}{l}\text { Gulshan-e- } \\
\text { Iqbal }\end{array}$ \\
\hline
\end{tabular}

Source: Police Record, 2014. 
HAND GRENADE ATTACK ON POLICE MOBILE NEAR SUZUKI SHOWROOM M.AJINNAH ROAD-2014: In the month of Nov 2014, Saad Aziz \& other accused persons attacked upon a police mobile with hand grenade resultantly police personnel namely (i) HC Shamsul Rehman \&(2) PC Mubeen embraced Shahadat whereas PC Faisal Eijaz was injured. The police mobile was completely damaged (burnt). Later on they informed me about this incident.

\begin{tabular}{|c|c|c|c|c|c|}
\hline S\# & FIR NO \& U/S & $\begin{array}{l}\text { DATE OF } \\
\text { OFFENCE }\end{array}$ & ARRESTED & $\begin{array}{l}\text { NOT } \\
\text { ARRESTED }\end{array}$ & $\begin{array}{l}\text { POLICE } \\
\text { STATION }\end{array}$ \\
\hline & $\begin{array}{l}\text { 690/2014 u/s } \\
\text { 353/324/302/ } \\
\text { 435/ PPC r/w } \\
\text { 3/4 Exp Act } \\
\text { r/w 7-ATA }\end{array}$ & $\begin{array}{l}10-11-14 \\
\text { at } 2000\end{array}$ & $\begin{array}{l}\text { i) Saad Aziz } \\
\text { (a) Tin Tin }\end{array}$ & $\begin{array}{l}\text { i) Umer@ } \\
\text { Hafiz@ } \\
\text { Jawad } \\
\text { ii) Ali } \\
\text { Rehman @ } \\
\text { Tona @ } \\
\text { Choto } \\
\text { iii) } \\
\text { Sharmeela } \\
\text { Pathan }\end{array}$ & Preedy \\
\hline
\end{tabular}

Source: Police Record, 2014.

ATTACK ON POLICE MOBILE OF BRIGADE PS-2014: In the year 2014, Saad Aziz \& other accused persons conducted in discriminate firing upon a police patrol car (white mehran) wherein police officials of Brigade PS were patrolling. Later on they informed me about this incident (In this incident ASI Arshad Tanoli embraced Shahadat whereas (1) PC Ghulam Aliof Brigade PSwas injured).

\begin{tabular}{|c|c|c|c|c|c|}
\hline S\# & $\begin{array}{l}\text { FIR NO \& } \\
\text { U/S }\end{array}$ & $\begin{array}{l}\text { DATE OF } \\
\text { OFFENCE }\end{array}$ & ARRESTED & $\begin{array}{l}\text { NOT } \\
\text { ARRESTED }\end{array}$ & $\begin{array}{l}\text { POLICE } \\
\text { STATION }\end{array}$ \\
\hline & $\begin{array}{l}249 / 2014 \mathrm{u} / \mathrm{s} \\
302 / 324 / 34 \\
\text { PPC r/w 7- } \\
\text { ATA }\end{array}$ & $\begin{array}{l}14-11-14 \\
\text { at } 0750 \\
\mathrm{hrs}\end{array}$ & $\begin{array}{l}\text { i) Saad Aziz } \\
\text { @ Tin Tin } \\
\text { ii) Asad-ur- } \\
\text { Rehman @ } \\
\text { Malik }\end{array}$ & $\begin{array}{l}\text { i) Umer @ } \\
\text { Haider } \\
\text { Abbas } \\
\text { ii) Ali } \\
\text { Rehman @ } \\
\text { Tona } \\
\text { Choto }\end{array}$ & Brigade \\
\hline
\end{tabular}

Source: Police Record, 2014.

HAND GRENADE ATTACK ON POLICE MOBILE NEAR AGS PAK BATTERY M.AJINNAH ROAD-2014: In the month of Nov 2014 same day after attack on police mobile of Brigade, Saad Aziz \& other accused persons again attacked upon a police mobile with hand 
grenade on M.A.Jinnah Road. In this incident police personnel namely HC Muhammad Asif was injured. Later on they informed me about this incident.

\begin{tabular}{|c|c|c|c|c|c|}
\hline S\# & FIR NO \& U/S & $\begin{array}{l}\text { DATE OF } \\
\text { OFFENCE }\end{array}$ & ARRESTED & $\begin{array}{l}\text { NOT } \\
\text { ARRESTED }\end{array}$ & $\begin{array}{l}\text { POLICE } \\
\text { STATION }\end{array}$ \\
\hline & $\begin{array}{l}705 / 2014 \mathrm{u} / \mathrm{s} \\
353 / 324 / 34 \\
\text { PPC r/w 3/4 } \\
\text { Exp Act r/w } \\
7- \\
\text { ATA }\end{array}$ & $\begin{array}{l}14-11- \\
14 \\
\text { at } 0755\end{array}$ & $\begin{array}{l}\text { i) Saad } \\
\text { Aziz@ } @ \text { Tin } \\
\text { Tin } \\
\text { ii) Asad- } \\
\text { ur-Rehman } \\
@ \\
\text { Malik }\end{array}$ & $\begin{array}{l}\text { i) Umer@ } \\
\text { Hafiz @ } \\
\text { Jawad } \\
\text { ii) Ali } \\
\text { Rehman@ } \\
\text { Tona } \\
\text { Choto }\end{array}$ & Preedy \\
\hline
\end{tabular}

HAND GRENADE ATTACK ON BECON HOUSE SCHOOL NORTH NAZIMABAD- 2015: In the month of March 2015, Saad Aziz $\&$ other accused persons hurled a hand grenade on Becon House School due to which the wall of school was damaged. However, there was no casualty or injury in this incident. Umer@ Haider Abbas also threw (ISIS) Daaishpamphlets at the place of incident.

\begin{tabular}{|l|l|l|l|l|l|}
\hline S\# & $\begin{array}{l}\text { FIR NO \& } \\
\text { U/S }\end{array}$ & $\begin{array}{l}\text { DATE OF } \\
\text { OFFENCE }\end{array}$ & ARRESTED & $\begin{array}{l}\text { NOT } \\
\text { ARRESTED }\end{array}$ & $\begin{array}{l}\text { POLICE } \\
\text { STATION }\end{array}$ \\
\hline & $53 / 2015$ & $18-03-15$ & i) Saad & i) Umer & North \\
& u/s & at 1032 & Aziz @ Tin & @ Hafiz & Nazimabad \\
& 3/4 Exp & & Tin & @ & \\
Act r/w 7- & & & Haider & \\
ATA & & & Abbas & \\
\hline
\end{tabular}

Source: Police Record, 2015.

FIRING UPON US NATIONAL Ms DEBRA LOBO-2015: In the month of April 2015, Saad Aziz@ @ Tin Tin and other accused persons fired upon a US Nation Ms Debra Lobo while she was driving her car at Bahadurabad Road. She was injured in this incident. Tayyab also thrown (ISIS) Da-Ish pamphlet at the place of incident. He was aware of the fact that Saad Aziz and his accomplices are going to target the above lady. 
Biannual Research Journal Grassroots Vol.55, No.I: 232-256

\begin{tabular}{|c|c|c|c|c|c|}
\hline S\# & $\begin{array}{l}\text { FIR NO \& } \\
\text { U/S }\end{array}$ & $\begin{array}{l}\text { DATE OF } \\
\text { OFFENCE }\end{array}$ & ARRESTED & $\begin{array}{l}\text { NOT } \\
\text { ARRESTED }\end{array}$ & $\begin{array}{l}\text { POLICE } \\
\text { STATION }\end{array}$ \\
\hline & $\begin{array}{l}273 / 2015 \\
\mathrm{u} / \mathrm{s} \quad 324 / 34 \\
\mathrm{r} / \mathrm{w} \text { 7-ATA }\end{array}$ & $\begin{array}{l}17-04-15 \\
\text { at } 1502\end{array}$ & $\begin{array}{l}\text { i) Saad } \\
\text { Aziz@ Tin } \\
\text { Tin }\end{array}$ & $\begin{array}{l}\text { i) Umer @ } \\
\text { HaiderAbbas } \\
\text { ii) All } \\
\text { Rehman @ } \\
\text { Tona @ } \\
\text { Choto } \\
\text { iii) Tayyab } \\
\text { iv) Mehmood }\end{array}$ & Ferozabad \\
\hline
\end{tabular}

Source: Police Record, 2015.

MURDER OF SABIN MEHMOOD (NGO WORKER) - 2015: In the month of April 2015, Saad Aziz@ @ Tin Tin and other accused persons fired upon and killed NGO worker Sabin Mehmood at Defence Phase 2, because she was using filthy language against Molana Abdul Aziz of Lal Masjid Islamabad. He was aware of the fact that Saad Aziz and his accomplices are going to kill the above lady.

\begin{tabular}{|l|l|l|l|l|l|}
\hline S\# & FIR NO \& U/S & $\begin{array}{l}\text { DATE OF } \\
\text { OFFENCE }\end{array}$ & ARRESTED & $\begin{array}{l}\text { NOT } \\
\text { ARRESTED }\end{array}$ & $\begin{array}{l}\text { POLICE } \\
\text { STATION }\end{array}$ \\
\hline & $214 / 2015$ & $25-04-15$ & i) Saad & i) Umer @ & Defence \\
u/s302/324/34 & at 2130 & Aziz @ & Haider Abbas & \\
& PPC r/w 7-ATA & Tin Tin & $\begin{array}{l}\text { ii) Ali Rehman } \\
\text { @ Tona @ }\end{array}$ & \\
& & & $\begin{array}{l}\text { Choto } \\
\text { iii) Tayyab } \\
\text { iv) Mehmood }\end{array}$ & \\
& & & & \\
& & & & \\
\end{tabular}

Source: Police Record, 2015.

MURDER OF MOCHI (SHOE REPAIRER)-2015: In the month of May 2015, after the incident of Safoora Goth a cobbler was killed by Saad Aziz@Tin Tin and other accused persons at DHA Karachi on suspicion of being informer of Police. He was aware of the fact that Saad Aziz and his accomplices are going to kill this person.

\begin{tabular}{|l|l|l|l|l|l|}
\hline S\# & $\begin{array}{l}\text { FIR NO \& } \\
\text { U/S }\end{array}$ & $\begin{array}{l}\text { DATE OF } \\
\text { OFFENCE }\end{array}$ & ARRESTED & $\begin{array}{l}\text { NOT } \\
\text { ARRESTED }\end{array}$ & $\begin{array}{l}\text { POLICE } \\
\text { STATION }\end{array}$ \\
\hline & $227 / 2015 \mathrm{u} / \mathrm{s}$ & $17-05-15$ & i) Saad Aziz & $\begin{array}{l}\text { i) Tayyab } \\
\text { ii) Mehmood }\end{array}$ & Gizri \\
& $324 / 34 \mathrm{PPC}$ & at 1930 & @ Tin Tin & ii) Mehmod \\
\hline
\end{tabular}

Source: Police Record.

NOTE: In all the above disclosed criminal activities and acts of terrorism the information and directives were being provided and 
issued by him (Tahir Minhas). Directly or indirectly he was connected with each and every case.

\begin{tabular}{|l|l|l|l|l|l|}
\hline S\# & FIR NO \& U/S & $\begin{array}{l}\text { DATE OF } \\
\text { OFFENCE }\end{array}$ & ARRESTED & $\begin{array}{l}\text { NOT } \\
\text { ARRESTED }\end{array}$ & $\begin{array}{l}\text { POLICE } \\
\text { STATION }\end{array}$ \\
\hline & $88 / 2015$ u/s & $20-05-$ & i) Tahir & i) Umer @ & CT\&FCIU, \\
& $353 / 324 / 427 / 34$ & 15 & Hussain & Haider & CTD Sindh, \\
& PPC, 3/4 Exp & at 0600 & Minhas @ & Abbas & Karachi. \\
Act r/w 7-ATA & to 0730 & Uncle @ & ii) Ali & \\
& & Sain Tona & Rehman @ & \\
& & ii) Saad & Tona @ & \\
& & Aziz @ Tin & Choto & \\
& & Tiin & iii) & \\
& & iii) Hafiz & Tayyab@ & \\
& & Nasir @ & Like Blue \\
& & Yasir & iv) Abdullah \\
iv) Azhar & Mansoori & \\
& & & & \\
& & & & & \\
& & & & &
\end{tabular}

PRESENT CRIMINAL RECORD OF CTD CIVIL LINE KARACHI

\begin{tabular}{|l|l|l|l|l|}
\hline S\# & FIR No. & \multicolumn{1}{|c|}{ U/s } & $\begin{array}{l}\text { DATE OF } \\
\text { OFFENCE }\end{array}$ & $\begin{array}{l}\text { POLICE } \\
\text { STATION }\end{array}$ \\
\hline 1 & $88 / 2015$ & $\begin{array}{l}353 / 324 / 427 / 34 \quad \text { PPC, } \\
\text { 3/4 Exp Act r/w 7-ATA }\end{array}$ & $\begin{array}{l}20-05- \\
2015\end{array}$ & $\begin{array}{l}\text { CT\&FCIU, } \\
\text { CTD }\end{array}$ \\
\hline 2 & $89 / 2015$ & $23-(\mathrm{i})$ A SAA & $20-05-2015$ & $\begin{array}{l}\text { CT\&FCIU, } \\
\text { CTD }\end{array}$ \\
\hline 3 & $88 / 2015$ & $\begin{array}{l}353 / 324 / 427 / 34 \text { PPC, 3/4 } \\
\text { Exp Act r/w7-ATA }\end{array}$ & $20-05-2015$ & $\begin{array}{l}\text { CT\&FCIU, } \\
\text { CTD }\end{array}$ \\
\hline 4 & $89 / 2015$ & 23-(i)/ASAA & $20-05-2015$ & $\begin{array}{l}\text { CT\&FCIU, } \\
\text { CTD }\end{array}$ \\
\hline
\end{tabular}

\section{CONCLUSION}

Terrorism is complex Phenomena which can only be tackled through reforming police and judicial system. Further co-operation between various agencies of law enforcement has become indispensable for dealing with terrorism.

Karachi's history is rampant with the incidents of terrorism. In most cases youth has been involved by certain elements. Acts of terrorism are sponsored by religious and political forces. International links have also been identified in this study.

Understaffing and lack of proper training have been identified as main factors for the lack of control over growing acts of terrorism in 
our country. Thus, reforming police, judicial system can enable us to eliminate terrorism.

\section{RECOMMENDATIONS}

From the analysis of primary data of accused of Safoora incident and secondary data of terrorism, the study recommends following:

1. Acts of terrorism in our country is linked to international terrorism. Therefore, there must be co-ordination and cooperation between various international agencies to contain the growing terrorism.

2. The one of the accused was arrested earlier but he was let off by the courts due to in sufficient evidence, therefore, there is need to effectively reform police, investigation and judicial system to bring the perpetrator to justice.

3. It was found in the study that all accused were Information Technology (IT) literate and highly qualified persons. They did not get education in Madrasah but got education in English medium Schools of Aand O level. Therefore, State should various measures to engage youth in positive activities. Government must take initiative to secure campuses by controlling various extremist forces.

4. State need to build and promote its counter extremism and counter terrorism narrative at educational institutions through conferences and seminars.

\section{REFERENCES}

Arif Hasan and Asiya Sadiq, (1996). Adapted in Laurent Gayer, Understanding Karachi, Based on data compiled by Arif Hasan and Asiya Sadiq for UNCHS Global Report on Human Settlements.

Asian Ethnicity Volume 4, Issue 2, (2003). Baloch Ethnic Nationalism in Pakistan: From Guerrilla War to Nowhere?

Central Police Office Karachi Sindh.

Crenshaw, M. (1992). Decisions to use terrorism: Psychological constraints on instrumental reasoning. In Social Movements and Violence: Participation in Underground Organizations, ed. D. D. Porta, Greenwich, London: JAI.

Crenshaw, M. (1998). The logic of terrorism: Terrorist behavior as a product of strategic choice. In Origins of Terrorism, ed. W. Reich, Washington, DC: Woodrow Wilson Center .

Crenshaw, M. 2000. The psychology of terrorism: An agenda for the $21^{\text {st }}$ century, Political Psychology 21(2):405-420.

Col. Waheed. Study on Karachi Violence. 
General Jahangir Karamat, (1999). Challenges and Opportunities for Pakistan, Henry L. Stimson Center Asian Security Luncheon Series, April 12, 1999.) p.85.

Ganor, B. (2005). The Counter-Terrorism Puzzle: A Guide for Decision Makers. New Brunswick, NJ: Transaction Publishers

Ganor, B. (2005b). The feasibility of post-modern terrorism. In Post Modern Terrorism, ed. B.

Ganor, Israel: The Interdisciplinary Center - the International Policy Institute for Counterterrorism.

Hoffman, B. (1995). Holy terror: The implications of terrorism motivated by areligious imperative. Studies in Conflict \& Terrorism 18(4):271-284.

Hoffman, B. (1998). Inside Terrorism. New York: Columbia University Press.

Hoffman, B. (2004). Defining terrorism. In Terrorism and Counterterrorism, eds. R.D. Howardand R. L. Sawyer, Dubuque: McGraw-Hill.

Hoffman, B. (2006). Inside Terrorism, Revised and Expanded Edition. New York: Columbia University Press.

Hoffman, B., and J. M. Taw. (1992). A Strategic Framework for Countering Terrorism and Insurgency, a Rand Note; N-3506-DOS. Santa Monica, CA.

Husain, Syed Ejaz, Terrorism In Pakistan: Incident Patterns, Terrorists' characteristics, And The Impact Of Terrorist arrests On Terrorism (2010). Publicly accessible Penn Dissertations. Paper 136.orism Incidents in Pakistan (1974-2007) (p.26).

Horgan, J. (2005). The Psychology of Terrorism. London: Routledge.

Millon, (1981). Masters of the Mind and Resolving different clinical syndrome

Mujtaba, (2009). Profiling of Karachi Violence Conflict and Peace Studies, Volume 2, Number 3.

Shabana Fayyaz, (1999). Pakistan Response Towards Terrorism, A Case Study of Musharraf Regime. 